\title{
NETWORKING-HUMAN AREA NETWORK
}

\author{
Tehseen Bano Mohd Anis ${ }^{1}$, Subha Subramaniam ${ }^{2}$ \\ ${ }^{I}$ Dept. of Electronics, Shah \& Anchor Kutchhi Engineering College, Mumbai, India \\ ${ }^{2}$ Dept. of Electronics, Shah \& Anchor Kutchhi Engineering College, Mumbai, India
}

\begin{abstract}
This paper grabs the detail study on information about Computer Networking. Two distinct electronic media communicating between themselves and forming a group within its area can be termed as Computer Networking. In the modern globe, communication is the most essential part of living creatures and the need of communication, from one end of the world to the other end, gave rise to the system of Networking. There are various types of forming of the network. Networking and its different types and uses will be studied throughout the paper.
\end{abstract}

Further part of the paper will deal in detail on the topic of networking devices that forms the base of a successful network. We propose a methodology to use human body as a transmitting medium to pass data in a Human Area Network (HAN).

Keywords-Computer Networking; communication; devices; Human Area Network (HAN). $* * *$

\section{INTRODUCTION}

The word Network in simple term means a group of devices coming together to share same or different information among themselves or to a specific device through a particular route. Such a scenario can be termed as a Network. Now a computer or any electronic device involved in these type of data sharing or information sharing can be coined as Computer Network. A computer network which in sometime also called as data network is nothing but a telecommunication setup that inspects computer to exchange information among them. Every networked computing equipment pass data to each other through data connections in Computer network. Transferring of data in Computer World is done in the form of Packets. For joining of the two devices, or in network language called as nodes, are connected either using physical cable media or through wireless media. The most common example of every day Computer Network usage is Internet.

Nodes are those computer devices that start, routes and terminates the data for a successful data transfer to takes place. Nodes can be anything such as PC, mobile phone, severs and any networking hardware. Any two devices whether they are connected directly or they have any intermediate between them, as long as they are able to exchange information or data amongst each other they are said to be networked together.

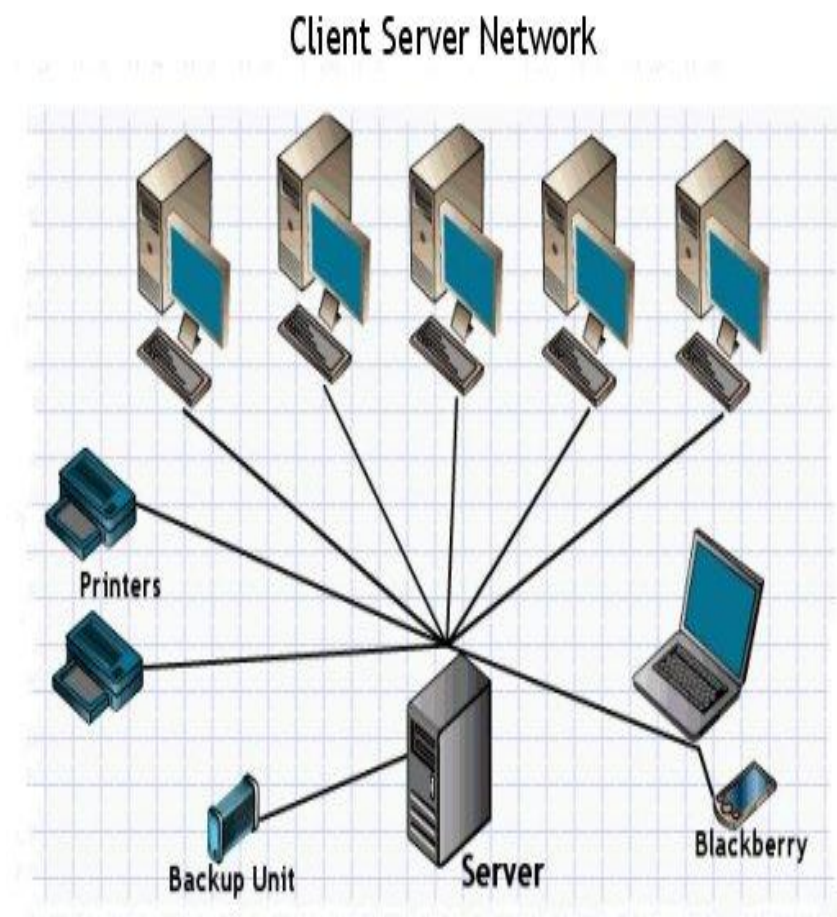

Fig 1

A computer network to transmit data signals differs in physical media, the protocols used for network traffic, network's size, topology used, etc. The physical layer is the layer that deals directly with physical media i.e. to the device through its connector or port. There are various and numerous applications of computer networks such as access to World Wide Web, printers, fax, emails, mobile and instant messaging applications.

Network is usually a form of data transfer across the world like internet and at the same time printing data in a small office. So the type of network depends on the area of usage. 


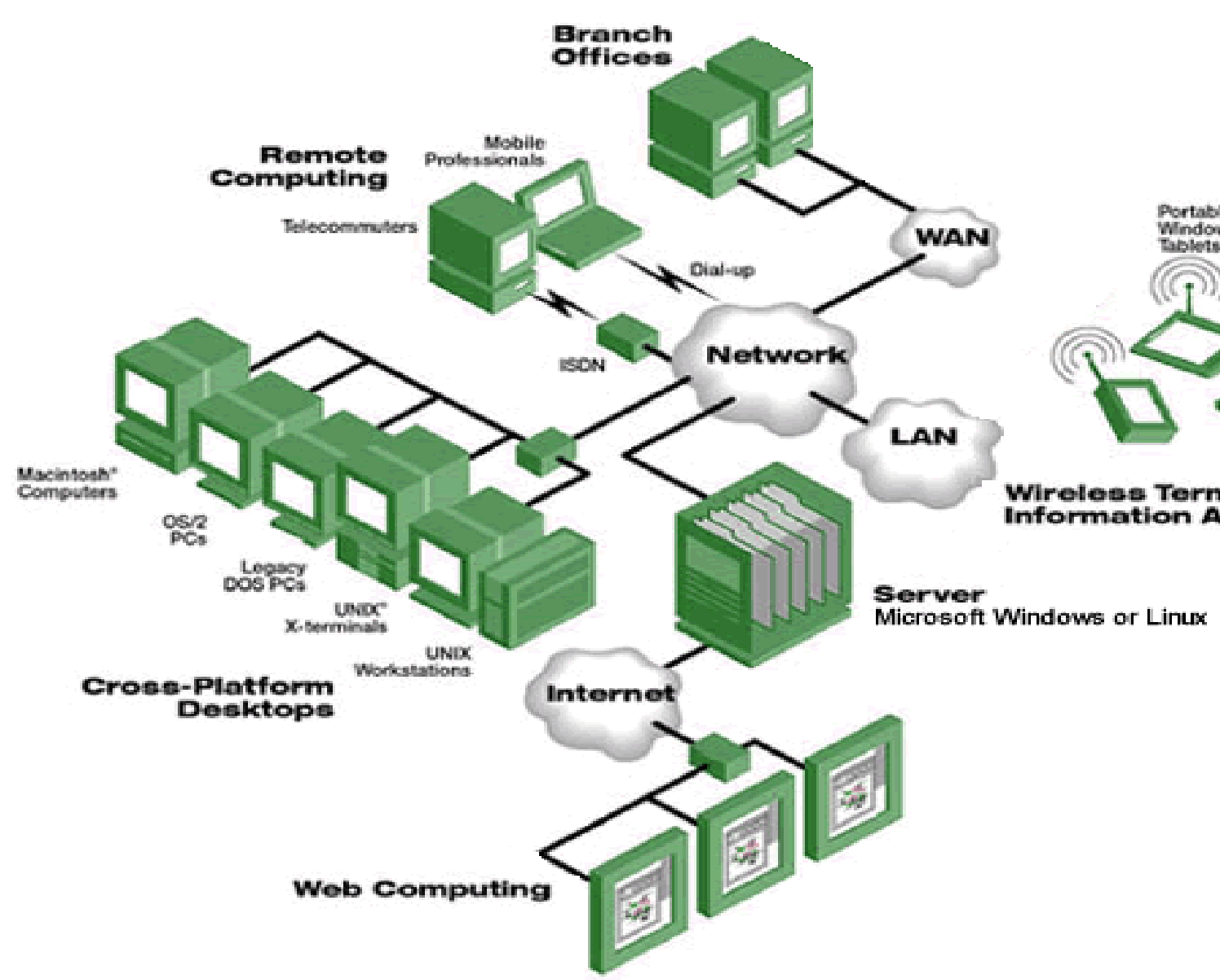

Fig 2: Various Network Types

There are several types of network in the current world we will look at the main of them. The list includes:

- $\quad$ LAN - Local Area Network

- WAN - Wide Area Network

- $\quad$ MAN - Metropolitan Area Network

- WLAN - Wireless Local Area Network

- HAN - Human Area Network

- $\quad$ PAN - Personal Area Network

LAN and WAN are the main and original types of area network and the rest were evolved in recent years according to the usage of it and the advancement of the technologies.

The two devices must communicate with each other about the accepting or transmitting data, this is done through certain protocols like TCP/IP protocols. Protocols are designed on the standard decided by International Organization for Standardization (ISO) with principle to Open Systems Interconnection (OSI) model.

In Human Area Network (HAN), which is the most personal used area network, the data is transferred with the touch of two human bodies. It is the most secured form of data transfer as the receiving end is very much clear and moreover the transferred data is not vulnerable to any network interference or data being hacked.

\section{LITERATURE REVIEW ON}

A network is a bunch of personal computers and other interconnected hardware components using communication channels that enables to access, exchange resources, data and information. Today's communication is interdependent on modern Computer networking techniques. All techniques involved in Public Switched Telephone Network (PSTN) are normally controlled by Computers. The main function behind the telephony is the Internet Protocol, not basically the public Internet. The visions of communication have boomed up by decades ago. And this could not have been possible without the advancement in the Computer Networks. It is Computer Networks and the involved techniques that allow communication to takes place between two distinct networked computers using softwares, networking devices, peripherals, etc.

In the initial time before the idea of network came into picture, for transfer or exchange of data it has to be done physically like we do with pen drive or compact disc. Physical sharing is possible with for small amount of work and that too within a specified area but it becomes very difficult where the sharing has to be done with millions of computers and the distance is quite far like Internet. This made the researchers to think and this gave rise to the idea of networking where the exchange of data or information can take place more efficiently. Hence, the initial network was formed. 
An organization called Homebrew Computer Club was founded by Lee Felsestein which includes lot of hackers from West Coach. In the late 1970, Lee worked on a project called Community Memory Project in which he actually worked on computer networks before the era of developed Personal Computers. This was a very basic system with just having a screen connected to a keyboard and this system was connected to a central processor which did the processing for it. This was setup in the Whole Earth Access store, Laundromats and community centers in San Francisco and this uses a permanent link over limited area.

In the field of networking, the initial early systems were Public Electronic Network (PEN) located in Santa Monica, the WELL (Whole Earth Lectronic Link) located in San Francisco, the Big Sky Telegraph, and small companies with online universities, etc. Gradually, in the late 1980, in the field of networking they also want to include Internet for networking and hence they took TCP/IP standard for it. It was then the Computer network was emerged with academic network for a boost in developing globally. The Public Electronic Network (PEN) located in Santa Monica was the first of its kind to build a network based local government of any size. It was run by the local government council members and the facility for the citizens include like forms, library access, city information and free email to keep in touch with the government. The Public Electronic Network (PEN) was started in February 1989.

Packet switching, a new type of protocol was introduced and it forms the basic principle of application like secure voice transmission in military. This technique soon became the leading protocol for the communication world in the $20^{\text {th }}$ century and with research and development these techniques were reformed into networks.

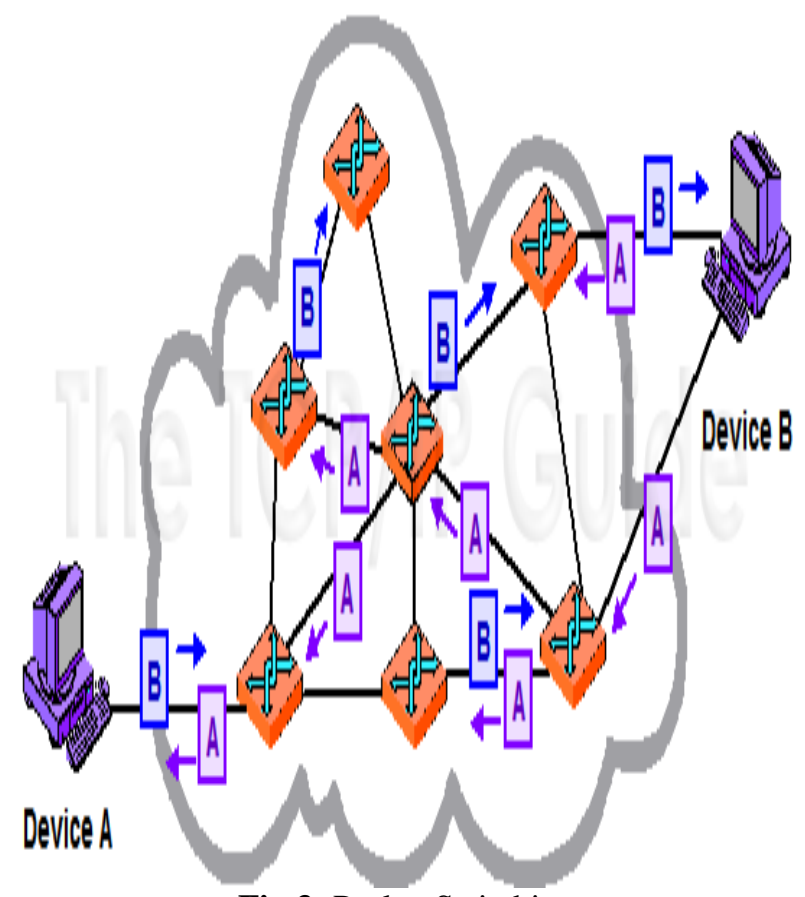

Fig 3: Packet Switching

\section{TYPES OF NETWORK}

\section{Global Wireless Standards}

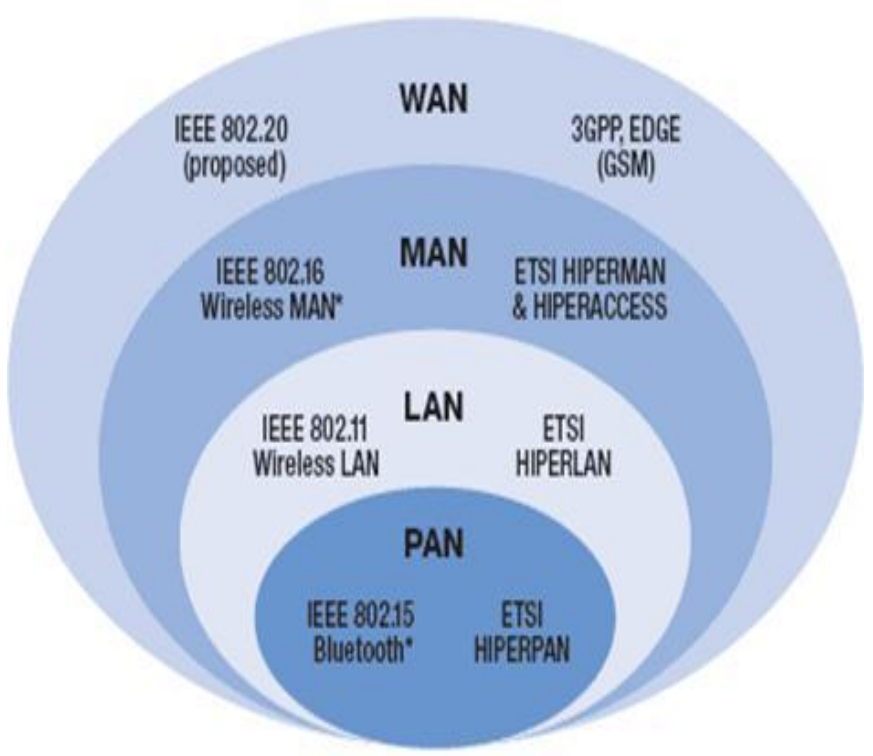

Fig 4: Types of Network

There are various types of networks that can be formed depending upon the area of interest like WAN, MAN, LAN, HAN, etc. Each is decided as how big or small the area, where the network is to be formed, is. The various types of network is discussed in detail:

\subsection{Local Area Network (LAN)}

Local Area Network is a network that connects computer or devices with each other within a limited range of areas such as home, school, office building, with using any network media. Standard technologies like token ring, ARCNET, etc. were used in the past but now LAN is build using most common techniques like Ethernet over twisted pair cabling and Wi-Fi.

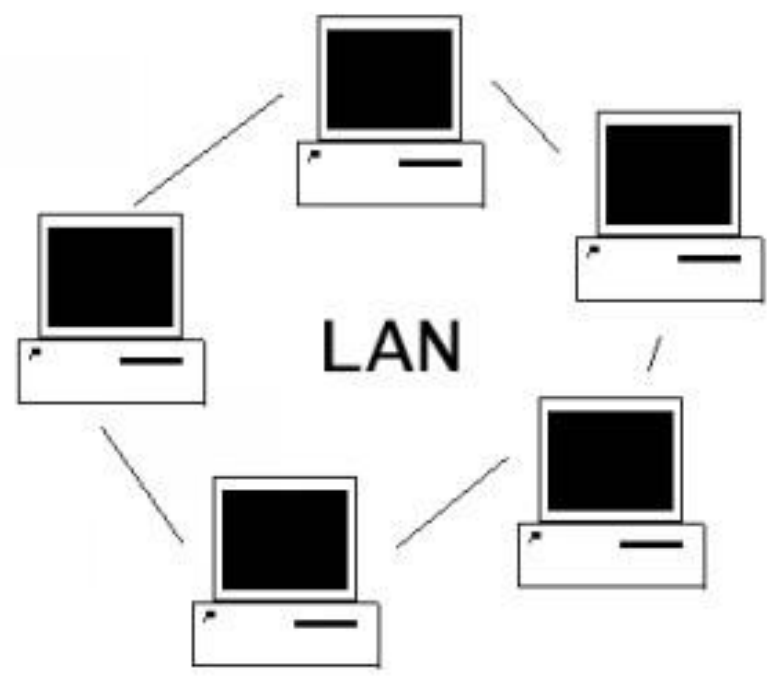

Fig 5: Local Area Network (LAN) 
Initially the LAN cabling was greatly dependent on the type of co-axial cable used. IBM's Token Ring LAN setup was done using shielded twisted pair cable. Then came the StarLAN with untwisted pair cable by utilizing Cat 3 cable that was also used for telephone cables. Hence, this gave birth to 10Base-T and its successors and proper structured cabling.

In today's world of wireless technologies through some techniques the wired cabling to build LAN setup is reduced to great extent. IEEE 802.11 is the IEEE standard for wireless LAN (WLAN). Most of the gadgets like mobile phones, laptops and computer comes up with built in wireless connectivity and hence it give rise to the need to be connected to network while moving. This makes Wireless Local Area Network of great usage. This is the most common network found in café, home, schools, etc.

The connection between two devices is shown by the network topologies whether it could be ring, bus, star or mesh. In LAN, at the physical and data link layers these type of topologies are used but the most common used now is switched Ethernet. And then as the layer rises it gets the TCP/IP as a standard protocol for transfer of data information. A simple LAN setup can have one or more switches which can be an ADSL modem for internet access, router or simply a cable modem. Switches, routers, firewalls, sensors, etc. all together forms a LAN. One LAN can be able to connect to another LAN with leased lines or via Internet using VPN. A LAN can be characterized as Metropolitan Area Network (MAN) or Wide Area Network (WAN) on the basis of secured connection and distance involved.

\subsection{Wide Area Network (WAN)}

A network that covers metropolitan, regional, national or even international borders using telephone leased lines are termed to be Wide Area Network (WAN). This form communication is useful for long distance communication where government bodies or private firm need to communicate with their branches which are located in other part of the world. The can easily exchange data or information among their employees, client, buyer or suppliers. It helps to reduce the large distance between two communicating bodies and hence make the work to work smoothly.

WAN forms a master connection to interconnect many LANs or other types of network so and such that in a given location all the users using the computer are connected together to the users in other location. Individual WAN are particularly built for specific tasks and are also privately built. They are made with the help of leased lines and as the lines end a router is connected to connect the LAN on side to the LAN's router on other side. Circuit switching or Packet switching methods can also be used for WAN which are less costly than leased lines. TCP/IP protocol is used for transport and addressing. WANs used protocols like Packet over SONET/SDH, ATM and Frame Relay by service provider companies for transferring. X.25 is the grandfather of Frame Relay protocol which was an initial important protocol and many functions of X.25 are still in use today by Frame Relay protocol.

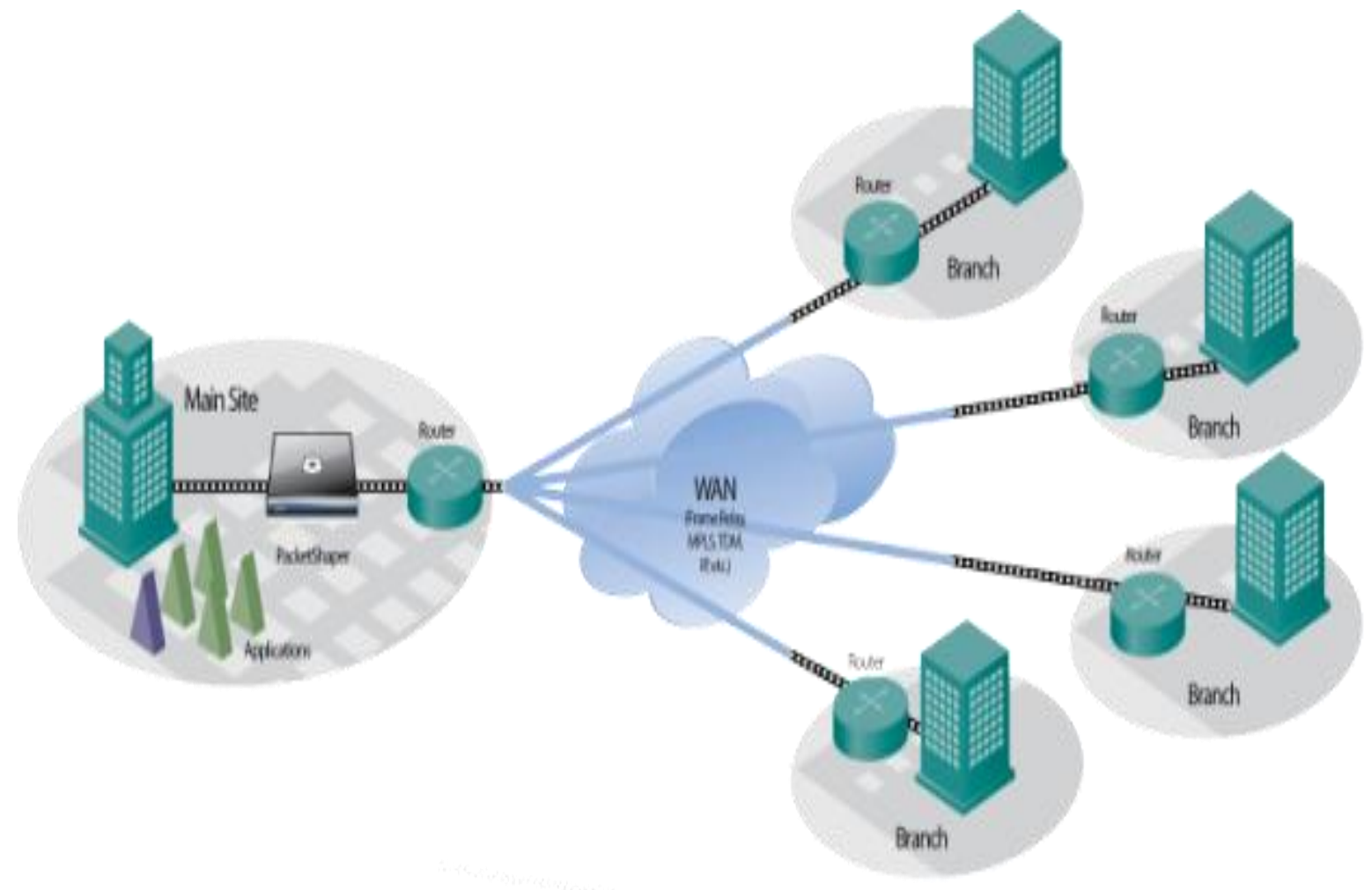

Fig 6: Wide Area Network (WAN) 


\subsection{Metropolitan Area Network (MAN)}

A Metropolitan Area Network is a network that connects a few places or blocks within a city. The area covered by Metropolitan Area Network (MAN) is larger than Local Area Network (LAN).

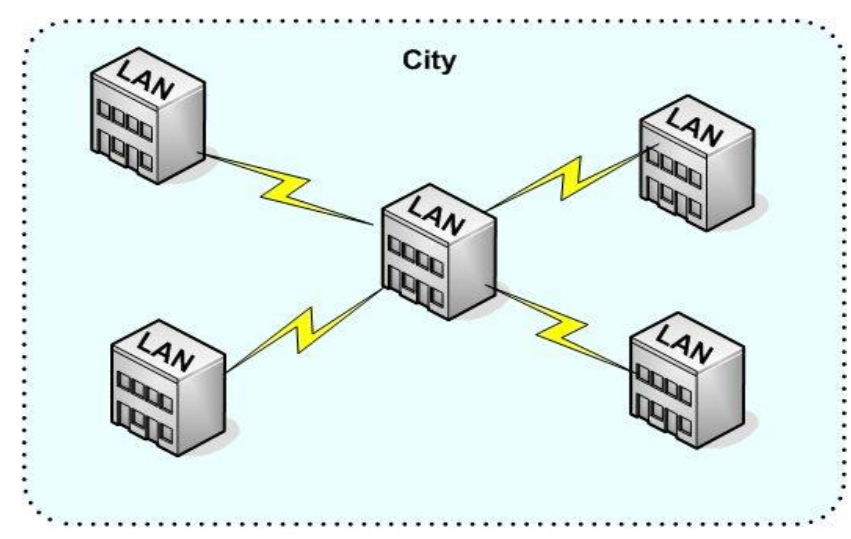

Metropolitan Area Network (MAN)

Fig 7: Metropolitan Area Network (MAN)

Kenneth C. Laudan and Jane P. Laudan define a metropolitan area network as:

"A Metropolitan Area Network (MAN) is a large computer network that spans a metropolitan area or campus. Its geographic scope falls between a WAN and LAN. MANs provide Internet connectivity for LANs in a metropolitan region, and connect them to wider area networks like the Internet."

It is also sometimes called as Municipal Area Network. The technologies used by this type of area network are SMDS, FDDI, and Asynchronous Transfer Mode (ATM). Though, now-a-days these technologies are replaced by Ethernet. The links between two or more LANs are connected using a MAN using wireless technologies like infrared transmission or radio waves. Many private companies take on rent these wireless services as applying long cables throughout could be a very expensive option.

In IEEE 802.6 standard, Distributed Queue Dual Bus (DQDB) is the standard maintained for Metropolitan Area Network. With this standard it enhances the network to spread along like 20 miles long and that too with an operating speed of about $34-155 \mathrm{Mbit} / \mathrm{s}$.

\subsection{Human Area Network (HAN)}

This is the newly network technology in which it uses the surface of the body for data transmission medium. When a transmitter is capacitively coupled to the human body and generates an AC field with a frequency lower than the resonant frequency of the body (approx. 70-100 MHz), the signal propagates over the surface of the body. Since it does not resonate with the body, the body does not act as an antenna. So, the signal is not radiated into the surroundings, but rather propagates over the surface and escapes to the Earth ground.

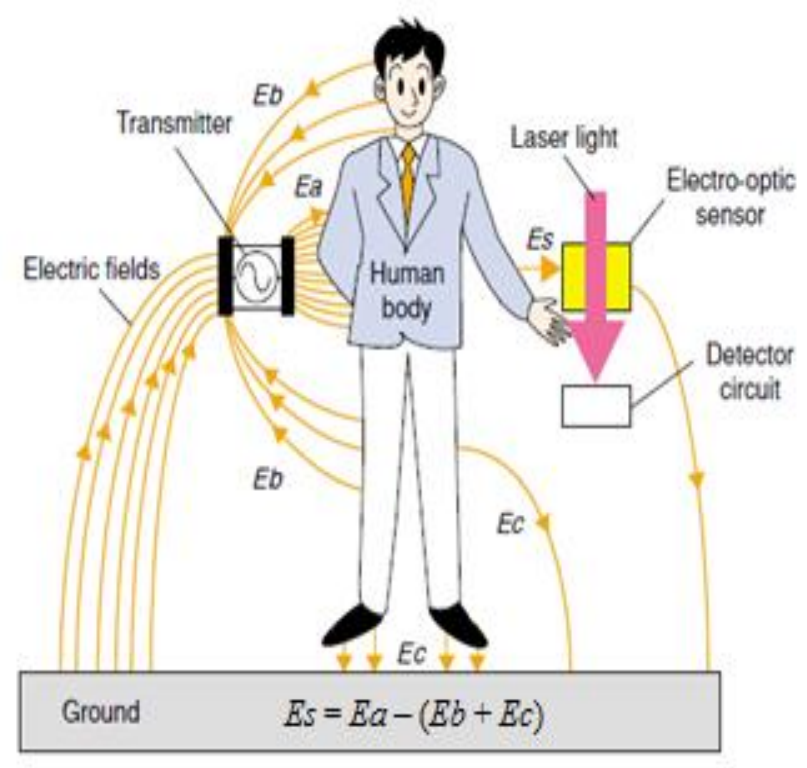

Fig 8: Human Area Network

In T. G. Zimmerman's electric-field model below the body is basically considered to be conductive, which means that the direction of the induced field is mostly perpendicular to the body. Moreover, bio-electromagnetic simulations have shown that almost none of the field penetrates the body. Clothing, the soles of shoes, and flooring can be considered capacitive and thus transparent to the AC signal.

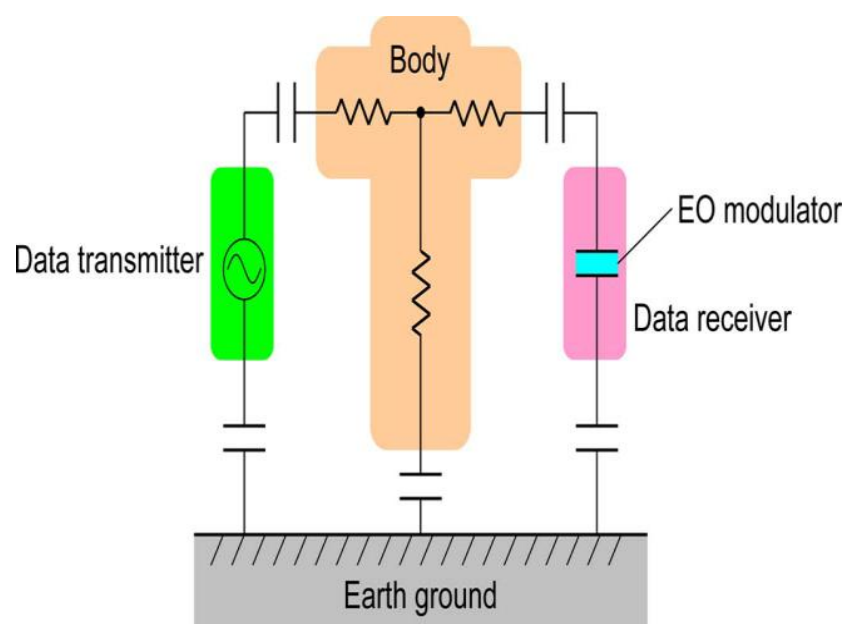

Fig 9: T. G. Zimmerman's electric-field model

\section{SOME NETWORKING DEVICES}

Bluetooth (over IEEE 802.15.1), ultra-wideband, ZigBee (over IEEE 802.15.4), and Wi-Fi (over IEEE 802.11) are three protocol standards for short range wireless communications with low power consumption and Human body as a medium.

\subsection{BLUETOOTH over IEEE 802.15.1}

Bluetooth, also known as the IEEE 802.15.1 standard is based on a wireless radio system designed for short-range and cheap devices to replace cables for computer 
peripherals, such as mice, keyboards, joysticks, and printers. This range of applications is known as wireless personal area network (WPAN). Two connectivity topologies are defined in Bluetooth: the piconet and scatternet. A piconet is a WPAN formed by a Bluetooth device serving as a master in the piconet and one or more Bluetooth devices serving as slaves.

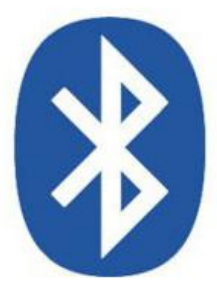

\section{Bluetooth}

Fig 10 Bluetooth

Slaves communicate only with their master in a point-topoint fashion under the control of the master. The master's transmissions may be either point-to-point or point-tomultipoint. Two piconets can be connected to form a scatternet. A Bluetooth device may participate in several piconets at the same time, thus allowing for the possibility that information could flow beyond the coverage area of the single piconet. A device in a scatternet could be a slave in several piconets, but master in only one of them.

\subsection{ZIGBEE over IEEE 802.15.4}

ZigBee over IEEE 802.15.4 defines specifications for low rate WPAN (LR-WPAN) for supporting simple devices that consume minimal power and typically operate in the personal operating space (POS) of $10 \mathrm{~m}$. ZigBee provides self-organized, multi-hop, and reliable mesh networking with long battery lifetime.

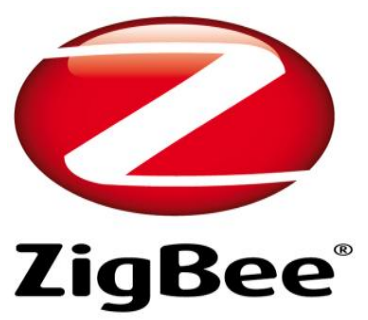

Fig 11: ZigBee

Two different device types can participate in an LR-WPAN network: a full-function device (FFD) and a reduced function device (RFD). The FFD can operate in three modes serving as a PAN coordinator, a coordinator, or a device. An FFD can talk to RFDs or other FFDs, while an RFD can talk only to an FFD. An RFD is intended for applications that are extremely simple, such as a light switch or a passive infrared sensor.
After an FFD is activated for the first time, it may establish its own network and become the PAN coordinator. All star networks operate independently from all other star networks currently in operation. Any of the FFDs may act as a coordinator and provide synchronization services to other devices or other coordinators. Only one of these coordinators can be the overall PAN coordinator, which may have greater computational resources than any other device in the PAN.

\subsection{Wi-Fi (Wireless Fidelity)}

Wireless fidelity (Wi-Fi) includes IEEE $802.11 \mathrm{a} / \mathrm{b} / \mathrm{g}$ standards for wireless local area networks (WLAN). It allows users to surf the Internet at broadband speeds when connected to an access point (AP) or in ad hoc mode. The IEEE 802.11 architecture consists of several components that interact to provide a wireless LAN that supports station mobility transparently to upper layers.

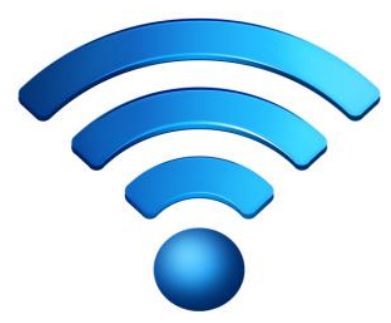

Fig 12: Wi-Fi

The basic cell of an IEEE 802.11 LAN is called a basic service set (BSS), which is a set of mobile or fixed stations. If a station moves out of its BSS, it can no longer directly communicate with other members of the BSS. Based on the BSS, IEEE 802.11 employs the independent basic service set (IBSS) and extended service set (ESS) network configurations. The IBSS operation is possible when IEEE 802.11 stations are able to communicate directly without any AP. Because this type of IEEE 802.11 LAN is often formed without pre-planning, for only as long as the LAN is needed, this type of operation is often referred to as an ad hoc network. Instead of existing independently, a BSS may also form a component of an extended form of network that is built with multiple BSSs. The architectural component used to interconnect BSSs is the distribution system (DS). The DS with APs allow IEEE 802.11 to create an ESS network of arbitrary size and complexity. This type of operation is often referred to as an infrastructure network. 


\begin{tabular}{|c|c|c|c|}
\hline Standard & Bluetooth & Zigbee & Wi-Fi \\
\hline IEEE spec.. & 802.15 .1 & 802.15 .4 & $802.11 \mathrm{a} / \mathrm{b} / \mathrm{g}$ \\
\hline Frequency band & $2.4 \mathrm{GHz}$ & $\begin{array}{c}868 / 915 \mathrm{MHz} \\
2.4 \mathrm{GHz}\end{array}$ & $2.4 \mathrm{GHz} ; 5 \mathrm{GHz}$ \\
\hline Max signal rate & $1 \mathrm{Mb} / \mathrm{s}$ & $250 \mathrm{~kb} / \mathrm{s}$ & $54 \mathrm{Mb} / \mathrm{s}$ \\
\hline Nominal range & $10 \mathrm{~m}$ & $10-100 \mathrm{~m}$ & $100 \mathrm{~m}$ \\
\hline $\begin{array}{c}\text { Nominal TX } \\
\text { power }\end{array}$ & $0-10 \mathrm{dBm}$ & $(-25)-0 \mathrm{dBm}$ & $15-20 \mathrm{dBm}$ \\
\hline $\begin{array}{c}\text { Number of RF } \\
\text { channels }\end{array}$ & 79 & $1 / 10 ; 16$ & $14(2.4 \mathrm{GHz})$ \\
\hline $\begin{array}{c}\text { Channel } \\
\text { bandwidth }\end{array}$ & $1 \mathrm{MHZ}$ & $\begin{array}{c}0.3 / 0.6 \mathrm{MHz} ; 2 \\
\mathrm{MHz}\end{array}$ & $22 \mathrm{MHz}$ \\
\hline Modulation type & GFSK & $\begin{array}{c}\text { BPSK (+ ASK) } \\
\text { 0-QPSK }\end{array}$ & $\begin{array}{c}\text { BPSK, QPSK } \\
\text { COFDM, CCK, M- } \\
\text { QAM } \\
\end{array}$ \\
\hline Spreading & FHSS & DSSS & $\begin{array}{c}\text { DSSS, CCK } \\
\text { OFDM }\end{array}$ \\
\hline $\begin{array}{l}\text { Coexistence } \\
\text { mechanism }\end{array}$ & $\begin{array}{l}\text { Adaptive freq. } \\
\text { hopping }\end{array}$ & $\begin{array}{c}\text { Dynamic freq. } \\
\text { selection }\end{array}$ & $\begin{array}{l}\text { Dynamic freq. } \\
\text { selection transmit } \\
\text { power control } \\
(802.11 \mathrm{~h})\end{array}$ \\
\hline Basic cell & Piconet & Star & BSS \\
\hline $\begin{array}{c}\text { Extension of the } \\
\text { basic cell }\end{array}$ & Scatternet & $\begin{array}{c}\text { Cluster tree- } \\
\text { mesh }\end{array}$ & ESS \\
\hline $\begin{array}{c}\text { Max number of } \\
\text { cell nodes } \\
\end{array}$ & 8 & $>65000$ & 2007 \\
\hline Data protection & 16-bit CRC & 16-bit CRC & 32-bit CRC \\
\hline
\end{tabular}

Fig 13: Comparison

\subsection{Human Body as a Transmission Medium}

Figure below shows a model of the electric-field distribution around a human body when an electric-field transmitter and receiver are attached to the body. The person stands on the Earth ground, and the transmitter and receiver have both signal and ground terminals. An AC field can be transmitted along the surface of the body as long as there is a capacitive connection. So the terminal is isolated with a layer of insulation, and no DC current flows into the body. Since the body is quite a good conductor, an AC electric field forms perpendicular to its surface. Communication is achieved when the receiver detects this AC field before it escapes to the Earth ground. However, part of the field induced on the body returns to the ground electrode of the transmitter, and a significant part escapes directly to the Earth ground. Furthermore, the field distribution changes continuously with a person's movements. Thus, the receiver must detect a signal that is quite faint and unstable. This presents an obstacle to obtaining good-quality transmission and to making the technology practical.
We first describe an equivalent circuit model that simplifies the basic approach to this problem. On the transmitter side, we need to address how to efficiently induce an AC electric field signal on the surface of the body. Both the human body and the transmitter are floating with respect to the Earth ground and they are capacitively-coupled. The capacitance tends to attenuate the $\mathrm{AC}$ signal produced by the transmitter.

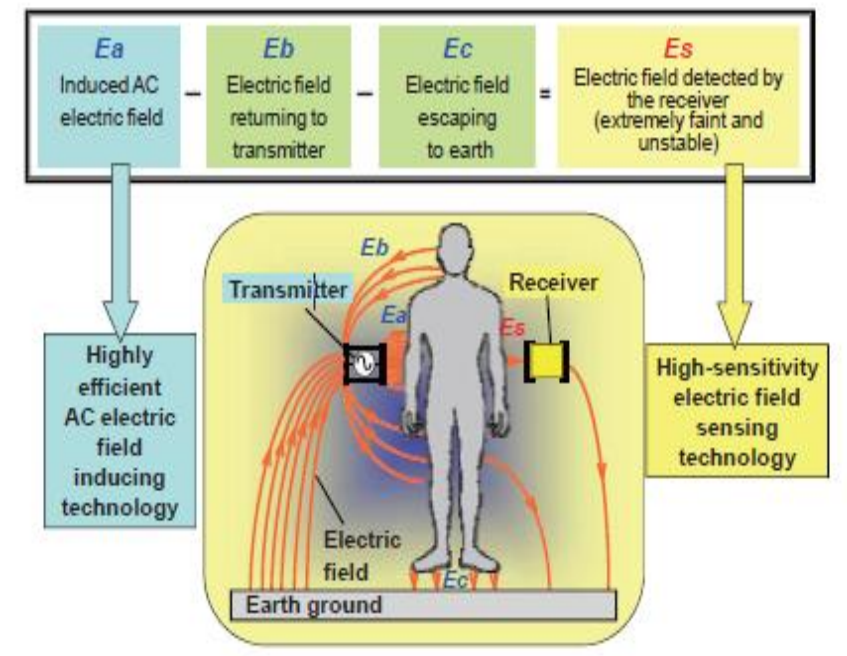

Fig 14: Human Body as a Medium

The capacitance of the connection also changes continuously as a person moves. To suppress the effects of changes in this capacitance, we developed technology involving the insertion of a reactance-matching circuit in the output stage of the transmitter. The strength of the induced signal is a maximum when the parasitic capacitance resonates with the reactance circuit.

\section{CONCLUSION}

This paper depicts the importance of computer network and its different setup structures. It gives the details about International Organization for Standardization (ISO) model called Open Systems Interconnection (OSI) model and its layers. OSI model forms the basis of any network. Various types of networks and some of networking devices are also studied. The paper deals with Human body as a transmitting device in Human Area Network (HAN). Thus, this paper gives various aspects of Computer Network in detail.

\section{REFERENCES}

[1] T. G. Zimmerman, "Personal Area Networks: Nearfield intra-body communication," IBM Systems Journal, Vol. 35, NOS 3\&4, pp. 609-617, 1996.

[2] IEEE Std 802-2002, IEEE Standard for Local and Metropolitan Area Networks: Overview and Architecture, page 1, section 1.2: "Key Concepts", "basic technologies"

[3] Karunakar Pothuganti and Anusha Chitneni "A Comparative Study of Wireless Protocols: Bluetooth, UWB, ZigBee, Wi-Fi”, Advance in Electronic and Electric Engineering, ISSN 22311297, Volume 4, Number 6 (2014), pp. 655-662 
[4] M. Shinagwa, et. al., "A near-field-sensing transceiver for intra-body communication based on the electro-optic effect," IEEE Trans. Instrum. Meas., Vol. 53, No. 6, pp. 1533-1538, 2004.

[5] Yuichi Kado, "Human-Area Networking Technology as a Universal Interface", 2009 Symposium on VLSI Circuits Digest of Technical Papers, 978-4-86348-010-0.

[6] YadongLi, Wenqiang Cui, DanIan Li, RuiZhang, "Research based on OSI model", 978-1-61284-4862/11/\$26.00 @2011 IEEE

[7] MENG Lin, MING Jun. Study on Understanding Manner of ISO/OSI Seven Layers Architecture [1]. Journal of Southwest Nationalities College Natural Science Edition, 2001, 27(1):35-39

[8] HUBERT ZIMMERMANN, "OS1 Reference Model-The IS0 Model of Architecture for Open Systems Interconnection", IEEE TRANSACTIONS ON COMMUNICATIONS, VOL. COM-28, NO. 4, APRIL 1980, 0090-6778/80/0400-0425\$00.75 0 1980 IEEE 